\title{
MicroRNA-218 Negatively Regulates Osteoclastogenic Differentiation by Repressing the Nuclear Factor-kB Signaling Pathway and Targeting Tumor Necrosis Factor Receptor 1
}

\author{
Weiwei Wang ${ }^{\mathrm{a}}$ Lei Yang ${ }^{\mathrm{b}} \quad$ Dan Zhang $^{\mathrm{a}}$ Chao Gao ${ }^{\mathrm{c}}$ Jie Wu \\ Yunxia Zhu ${ }^{d}$ Hongxiu Zhang ${ }^{\text {a }}$ \\ aDepartment of Obstetrics and Gynecology, The First Affiliated Hospital of Nanjing Medical University, \\ Nanjing, 'Department of Orthopedics, Nanjing First Hospital, Nanjing Medical University, Nanjing, \\ 'State Key Laboratory of Reproductive Medicine, Department of Obstetrics and Gynecology, The \\ First Affiliated Hospital of Nanjing Medical University, Nanjing, 'Key Laboratory of Human Functional \\ Genomics of Jiangsu Province, Jiangsu Diabetes Center, Nanjing Medical University, Nanjing, China
}

\section{Key Words}

Postmenopausal osteoporosis • MiR-218 • TNFR1 • NF-KB • Osteoclast differentiation

\begin{abstract}
Background/Aims: Postmenopausal osteoporosis is a common disease associated with estrogen deficiency leading to bone loss and bone tissue changes. The resultant bone fragility and increased risk of fracture has serious adverse effects on health and quality of life of the elderly, making it an important health issue. MicroRNA-218 (miR-218) is closely related to the development of osteoporosis. In this study, we investigated the regulatory mechanisms of miR-218 in osteoclastogenesis. Methods: We investigated miR-218 levels on differentiation of RAW 264.7 cells into osteoclasts compared with normal cells. Next, RAW 264.7 cells were transfected with miR-218 mimics or inhibitors to study the role of miR-218 in osteoclastogenic differentiation. Tartrate-resistant acid phosphatase (TRAP) staining was performed to determine osteoclastogenic differentiation. Bioinformatics analysis and luciferase reporter assay were used to identify and validate miR-218 target genes. Results: miR-218 was downregulated following RAW 264.7 cell differentiation into osteoclasts. miR-218 overexpression attenuated osteoclast differentiation, whereas low miR-218 expression promoted it as demonstrated by increased expression of osteoclast-specific genes and TRAP staining. Bioinformatics analysis and the luciferase reporter assay showed that tumor necrosis factor receptor 1 (TNFR1), a cell membrane receptor of TNF (TNF is an activator of nuclear factor- $\mathrm{kB}[\mathrm{NF}-\mathrm{kB}]$ ), is a direct

W. Wang and L. Yang contributed equally to this work.

\begin{tabular}{ll}
\hline Hogxiu Zhang, PhD, MD & Department of Obstetrics and Gynecology, First Affiliated Hospital of Nanjing Medical \\
& University, 300 Guangzhou Road, Nanjing 210029 (China) \\
& Tel. +86-25-86214093, Fax +86-25-86214093, E-Mail hongxiuz@njmu.edu.cn
\end{tabular}
\end{abstract}


target of miR-218. Conclusions: Our findings indicate that miR-218 regulates osteoclastogenic differentiation negatively by repressing NF-KB signaling by targeting TNFR1, suggesting that targeting miR-218 may be a therapeutic approach in postmenopausal osteoporosis.

(C) 2018 The Author(s)

Published by S. Karger AG, Basel

\section{Introduction}

Postmenopausal osteoporosis is a common disease characterized by reduced bone mineral density, bone architecture deterioration, and skeletal fragility. It leads to increased risk of fragility fracture and is mainly caused by disruption of the balance between osteoclast bone resorption and osteoblast bone formation [1-4].

Encoded by endogenous genes, microRNAs (miRNAs) are a class of noncoding singlestranded RNA molecules that are about 19-25 nucleotides long and that play an important regulatory role in various biological development processes [5]. miRNAs regulate the expression of their target genes mainly through post-transcriptional regulation of the genes, thereby participating in tumorigenesis, biological development, organogenesis, virus defense, and metabolism [6-8]. miRNAs also play roles in the occurrence and development of osteoporosis [9-13].

Research has focused on the association of miR-218 with bone formation [14-16]. For example, miR-218 can promote osteoblast differentiation by targeting the Wnt signaling pathway [14]. Another study suggested that miR-218 promotes human adipose tissuederived stem cell differentiation into osteoblasts [15]. Zhang et al. [16] suggested that miR218 regulated osteogenic differentiation by targeting the transcription factor runt-related transcription factor 2 (Runx2).

Expression of miR-218 is low in CD14+ peripheral blood mononuclear cells (PBMCs) in women with postmenopausal osteoporosis. PBMCs are osteoclast precursors [17]. However, the mechanism of miR-218 regulation of osteoclastogenesis requires further investigation. The aim of this study was to evaluate the effects of miR-218 on osteoclast differentiation and the potential molecular mechanism.

\section{Materials and Methods}

Osteoclastogenic Differentiation of RAW 264.7 Cells

The RAW 264.7 cell line, a mouse osteoclast precursor cell line, was obtained from FuHeng Cell Center (Shanghai, China) for the osteoclastogenesis studies. The cells were stimulated with receptor activator for nuclear factor- $\kappa$ B ligand (RANKL) (50 ng/mL: R\&D Systems, Minneapolis, MN) for 0, 1, 3, and 5 days to induce osteoclast differentiation. The cells were maintained under standard cell culture conditions $\left(5 \% \mathrm{CO}_{2}\right.$ and 95\% humidity). Osteoclasts induced in culture were assessed by tartrate-resistant acid phosphatase (TRAP) staining and osteoclast differentiation marker genes (i.e., tumor necrosis factor receptor-associated factor 6 [Traf6] and nuclear factor of activated T cells 1 [Nfatc1] mRNA. TRAP-positive multinuclear osteoclasts (>3 nuclei) were counted under light microscopy.

\section{miRNA Mimic/Inhibitor Transfection}

Fluorescence-tagged miR-218 mimic, inhibitor, and negative control (miR-NC) were synthesized by RiboBio Co., Ltd., (Guangzhou, China). For transfection, RAW 264.7 cells were seeded in 6-well plates (20 x $10^{4}$ cells/well) and transfected with $100 \mathrm{nM}$ miR-218 mimic and $150 \mathrm{nM}$ miR-218 inhibitor or miR-NC using Lipofectamine ${ }^{\circledR} 3000$ (Life Technologies, Houston, TX). After a 36 h incubation, transfection efficiency was observed under fluorescence microscopy. Then, the cells were cultured in fresh medium and incubated with RANKL for 3 days.

miRNA Extraction

Total RNA was extracted from the collected cells at $0,1,3$, and 5 days of differentiation using TRIzol (Ambion by Life Technologies, Camarillo, CA). Briefly, the cells were collected in an Eppendorf tube (EP tube), 


\section{Cellular Physiology Cell Physiol Biochem 2018;48:339-347 \begin{tabular}{l|l} 
DOI: 10.1159/000491740 & $\begin{array}{l}\text { O 2018 The Author(s). Published by S. Karger AG, Basel } \\
\text { www.karger.com/cpb }\end{array}$
\end{tabular} \\ Wang et al.: MiR-218 Downregulation of Osteoclastogenesis}

lysed with $1 \mathrm{~mL} /$ tube TRIzol, and then mixed with chloroform ( $0.2 \mathrm{~mL}$ TRIzol reagent). After centrifugation at $12,000 \mathrm{rpm}$ at $4^{\circ} \mathrm{C}$ for $15 \mathrm{~min}$, the supernatant was decanted to a new EP tube and mixed with cold isopropanol ( $0.5 \mathrm{~mL}$ TRIzol reagent). The mixture was then centrifuged at $12,000 \mathrm{rpm}$ at $4^{\circ} \mathrm{C}$ for $10 \mathrm{~min}$; the upper aqueous phase was discarded, and the RNA precipitate was collected and washed twice in $75 \%$ ethanol then centrifuged at $12,000 \mathrm{rpm}$ at $4^{\circ} \mathrm{C}$ for $15 \mathrm{~min}$. After removing the upper aqueous phase, the RNA precipitate was air-dried at room temperature, and 20-50 $\mu \mathrm{L}$ diethylpyrocarbonate-treated water was added to each tube to dissolve the precipitate before the total RNA was subjected to reverse transcription quantitative PCR (RT-qPCR) analysis.

\section{$R T-q P C R$}

Total RNA was isolated using TRIzol reagent. Reverse transcription was conducted using a PrimeScript RT reagent kit (TaKaRa, Shiga, Japan). Real-time RT-PCR specific primers for mouse miR218 and the internal reference U6 were from Sangon Biotech Co., Ltd., (Shanghai, China). The following primers were used for RT-qPCR detection: U6 (forward: 5'-AGAGAAGATTAGCATGGCCCCTG-3', reverse: 5 '-ATCCAGTGCAGGGTCCGAGG-3'); miR-218 (forward: 5'-TTGCGGATGGTTCCGTCAAGCA-3', reverse: $\quad 5^{\prime}$-ATCCAGTGCAGGGTCCGAGG-3'); Tnfr1 (forward: 5'-GGGGATACATCCATCAGGGGT-3', reverse: $5^{\prime}$-GCTCGGACAGTCACTCACC-3'); Nfatc1 (forward: $5^{\prime}$-GACCCGGAGTTCGACTTCG-3', reverse: 5'-TGACACTAGGGGACACATAACTG-3'); Traf6 (forward: 5'-AAAGCGAGAGATTCTTTCCCTG-3', reverse: 5'-ACTGGGGACAATTCACTAGAGC-3'); glyceraldehyde-3-phosphate dehydrogenase (Gapdh, forward: 5'-TGGCCTTCCGTGTTCCTAC-3', reverse: 5'-GAGTTGCTGTTGAAGTCGCA-3'). Amplification and detection were performed using the SYBR Premix Ex Taq II kit (TaKaRa) and Applied Biosystems StepOnePlus RealTime PCR System (Thermo Fisher Scientific, Waltham, MA), respectively. U6 and Gapdh were used as internal references.

\section{Target Gene Prediction}

We predicted the potential target genes of miR-218 using TargetScan, miRanda, and PicTar. We discovered that among the candidate target genes, the $3^{\prime}$ untranslated region ( $3^{\prime}$ UTR) of Tnfr1, which is closely correlated with the osteoclast signaling pathway, has an miR-218-binding site.

\section{Luciferase Reporter Assay}

The luciferase reporter vector of the Tnfr1 3' UTR was obtained from GeneChem Co., Ltd., (Shanghai, China). Site-directed mutagenesis was performed using the Stratagene QuikChange Lightning Site-Directed Mutagenesis Kit (Agilent Technologies, Santa Clara, CA). To detect suppression by miR-218, HEK293T cells were co-transfected with the indicated Tnfr $13^{\prime}$ UTR luciferase reporter (with either wild-type [WT] or mutant miR-218 binding sites). Renilla luciferase was transfected as efficiency control. Luciferase activity was measured using a luciferase assay system (Promega, Fitchburg, WI) according to the manufacturer's instructions.

\section{Western Blotting}

The cells were lysed with cell lysis buffer and protein concentration was measured using a bicinchoninic acid protein assay kit (Beyotime, Beijing, China) according to the manufacturer's protocols. Total protein $(80 \mu \mathrm{g})$ was boiled with $1 \times$ loading buffer for $5 \mathrm{~min}$, placed on ice, resolved with $10 \%$ sodium dodecyl sulfate-polyacrylamide gel electrophoresis, and then transferred onto polyvinylidene fluoride membranes (Biosharp Co., Ltd., Hefei, China). The membranes were blocked in $5 \%$ fat-free milk at $4^{\circ} \mathrm{C}$ for $1 \mathrm{~h}$ and then incubated overnight with primary antibody (NFATC1, TRAF6, TNFR1, p65, p-p65, GAPDH) at $4^{\circ} \mathrm{C}$. After removing unbound antibody and washing with Tris-buffered saline and Tween 20 (TBST) buffer three times, the membranes were incubated with horseradish peroxidase-conjugated secondary antibody for $1 \mathrm{~h}$ at $37^{\circ} \mathrm{C}$, and then washed with TBST buffer three times. Membranes were developed with a Tanon $4200 \mathrm{SF}$ automated fluorescence chemiluminescence image analysis system (Tanon, Shanghai, China).

\section{TRAP Staining}

Osteoclasts were confirmed using a TRAP staining kit according to the manufacturer's protocols (Sigma, St. Louis, MO). Briefly, cells were fixed with stationary liquid for $30 \mathrm{~s}$ at room temperature, and then washed with deionized water three times. To prepare the staining solution, the following was combined in a 
$100 \mathrm{~mL}$ beaker: $45 \mathrm{~mL}$ deionized water $\left(37^{\circ} \mathrm{C}\right), 1 \mathrm{~mL}$ combined Fast Garnet GBC base solution ( $\left.0.5 \mathrm{~mL}\right)$, and sodium nitrite solution ( $0.5 \mathrm{~mL}$ ), $0.5 \mathrm{~mL}$ naphthol AS-BI phosphate solution, $2 \mathrm{~mL}$ acetate solution, and $1 \mathrm{~mL}$ tartrate solution. The cells were immersed in the staining solution and incubated at $37^{\circ} \mathrm{C}$ for $1 \mathrm{~h}$, and then washed with deionized water. TRAP-positive cells (containing $>3$ nuclei) were observed under microscopy.

\section{Statistical Analysis}

All data are reported as means \pm standard deviations. Comparison between groups was done using one-way analysis of variance, followed by post-hoc testing (least significant difference). $P<0.05$ was used for significance. All experiments were performed in triplicate.

\section{Results}

\section{miR-218 was Downregulated in Osteoclastogenesis}

We monitored miR-218 expression at $0,1,3$, and 5 days after induction with RANKL; day 0 was considered negative control. As expected, RANKL induced marked osteoclastic differentiation in the RAW 264.7 cells, as evidenced by enhanced TRAP staining [18] (Fig. 1A), and increased mRNA and protein expression of NFATC1 and TRAF6, which are markers of mature osteoclasts $[19,20]$ (Fig. 1B, C). Then, we used qRT-PCR to detect miR-218 in osteoclastogenesis, and found that miR-218 expression was significantly reduced after osteoclastic differentiation, and miR-218 levels were lowest at 5 days after induction (Fig. 1D). These results suggest that miR-218 is downregulated in osteoclastogenesis.

\section{miR-218 Upregulation Inhibited Osteoclast Differentiation}

To explore the role of miR-218 in osteoclast differentiation, RAW 264.7 cells were transfected with miR-218 mimics, inhibitors, or miR-NC. As expected, transfection efficiency was $>60 \%$ in all three groups (Fig. 2A). miR-218 mimics induced significant elevation of miR-218 expression (nearly 228-fold of the miR-NC group) and the inhibitors markedly reduced miR-218 expression (nearly one-fifth of the miR-NC group) (Fig. 2B). TRAP staining showed that miR-218 overexpression weakened the effects of RANKL on osteoclastogenesis (Fig. 2C). However, miR-218 inhibition promoted osteoclast differentiation. qPCR and western blotting were performed to evaluate NFATC1 and TRAF6 expression profiles in the miR-218 mimics, miR-218 inhibitors, and miR-NC groups. miR-218 upregulation markedly inhibited osteoclast differentiation, as demonstrated by decreased expression of the osteoclast-specific genes Nfatc1 and Traf6 (Fig. 2D, E). However, low miR-218 expression significantly stimulated RAW 264.7 cell osteoclastic differentiation, which is consistent with the abovementioned results. Taken together, these data show that miR-218 upregulation attenuates osteoclast differentiation.

Fig. 1. miR-218 was downregulated in osteoclastogenesis. (A) TRAP staining confirmed osteoclast differentiation at 1,3 , and 5 days during osteoclastogenesis. Scale bar $50 \mu \mathrm{m}$. (B) Nfatc1 and Traf6 mRNA expression at 1, 3, and 5 days during osteoclastogenesis. (C) NFATC1 and TRAF6 protein expression at 1, 3 and 5 days during osteoclastogenesis. (D) miR-218 expression at 1,3 , and 5 days during osteoclastogenesis. $n=3$ for the experiments $\left({ }^{*} \mathrm{p}<0.05\right.$ compared with control).

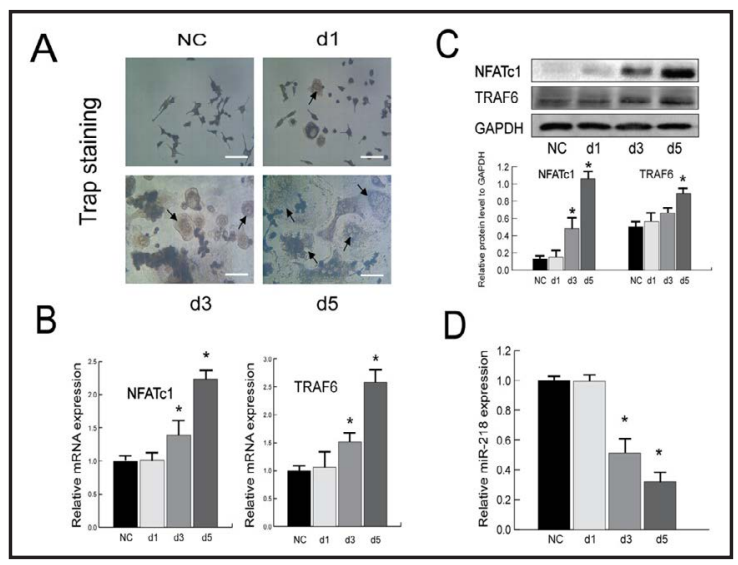


Fig. 2. miR-218 inhibited osteoclast differentiation. (A) Transfection efficiency was observed by light microscopy (left) (Scale bar $10 \mu \mathrm{m}$ ), Cells transfected with fluorescent blots were regarded as successfully transfected cells and transfection efficiency was expressed as follows: successfully transfected cells / total cells (right). (B) Relative miR-218 expression in RAW 264.7 cells transfected with miR-218 mimics, inhibitors, or miR-NC was detected by qRT-PCR to show the effects of transfected mimics and inhibitors in cells. (C) TRAP staining in RAW 264.7 cells expressing miR218 mimics, inhibitors, and miR-NC after 3-day RANKL induction. Scale bar $50 \mu \mathrm{m}$. (D) qRT-PCR of the Nfatc1 and Traf6 in cells expressing miR218 mimics, miR-218 inhibitors, and miR-NC. (E) Western blotting of NFATC1 and TRAF6 in cells expressing miR-218 mimics, inhibitors, and miRNC. $\mathrm{n}=3$ for the experiments $\left({ }^{*} \mathrm{p}<0.05\right.$ compared with miR-NC).

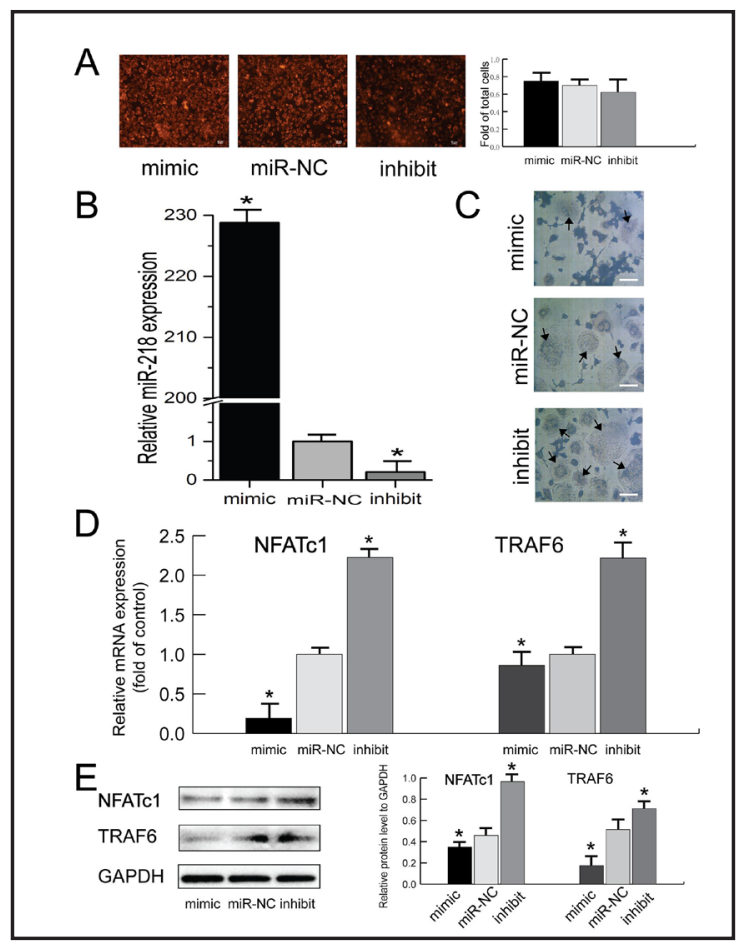

Fig. 3. TNFR1 was a direct target of miR-218. (A) TargetScan, miRanda, and PicTar were used to predict the potential target genes that stimulate osteoclast differentiation. (B) Schematic representation showing that the Tnfr $13^{\prime}$ UTR has a miR-218 binding site. (C) HEK293T cells were co-transfected with the Tnfr1 $3^{\prime}$ UTR luciferase reporter (either WT or mutant [Mut] miR-218 binding sites); miR-218 significantly inhibited the

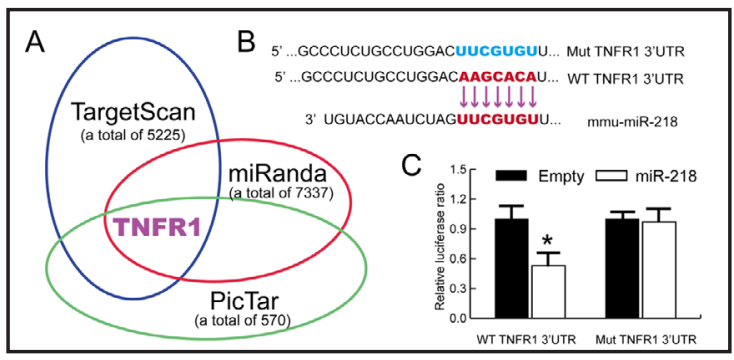
luciferase activity of WT Tnfr $13^{\prime}$ UTR. $n=3$ for the experiments $\left({ }^{*} \mathrm{p}<0.05\right.$ compared with the empty vector group).

\section{TNFR1 was a Direct Target of miR-218}

To investigate the potential molecular mechanism of miR-218 regulation of RAW 264.7 cell osteoclastic differentiation, we used TargetScan, miRanda, and PicTar to identify potential target genes that stimulate osteoclast differentiation (Fig. 3A). Among the candidate osteoclast-related target genes (e.g., c-Jun $\mathrm{N}$-terminal kinase (JNK), phosphoinositide 3 -kinase, transforming growth factor- $\beta$-activated kinase 1 (TAK1), pyruvate dehydrogenase kinase 1, RIAK4), we found that the Tnfr1 3' UTR has a miR-218 binding site (Fig. 3B). To investigate whether miR-218 could decrease Tnfr1 expression by binding its $3^{\prime}$ UTR, HEK293T cells were co-transfected with the Tnfr1 3' UTR luciferase reporter vector along with a vector expressing miR-218. Then, the Tnfr1 seed region for miR-218 binding was mutated and the luciferase assay was used to determine whether the observed changes were due to miR-218 recognition of its predicted binding site in Tnfr1 rather than non-specific effects. miR-218 significantly inhibited WT Tnfr1, but not mutant Tnfr1 (Fig. 3C), suggesting that Tnfr1 is a direct target of miR-218.

\section{miR-218 Inhibited Activation of p65 Signaling}

To further explore the molecular mechanism underlying miR-218 inhibition of osteoclastogenesis, we analyzed the potential molecules in the TNFR1 signaling pathway. To 
Fig. 4. miR-218 inhibited p65 signaling activation. (A) Western blotting of TNFR1, p65, and p-p65 at $0,1,3$, and 5 days during osteoclastogenesis $\quad\left({ }^{*} \mathrm{p}<0.05\right.$ compared with d0 group). (B) Western blotting of TNFR1, p65 and p-p65 in cells expressing miR218 mimics, inhibitors, and miRNC during osteoclastogenesis. $\mathrm{n}$ $=3$ for the experiments $\left({ }^{*} \mathrm{p}<0.05\right.$ compared with miR-NC).

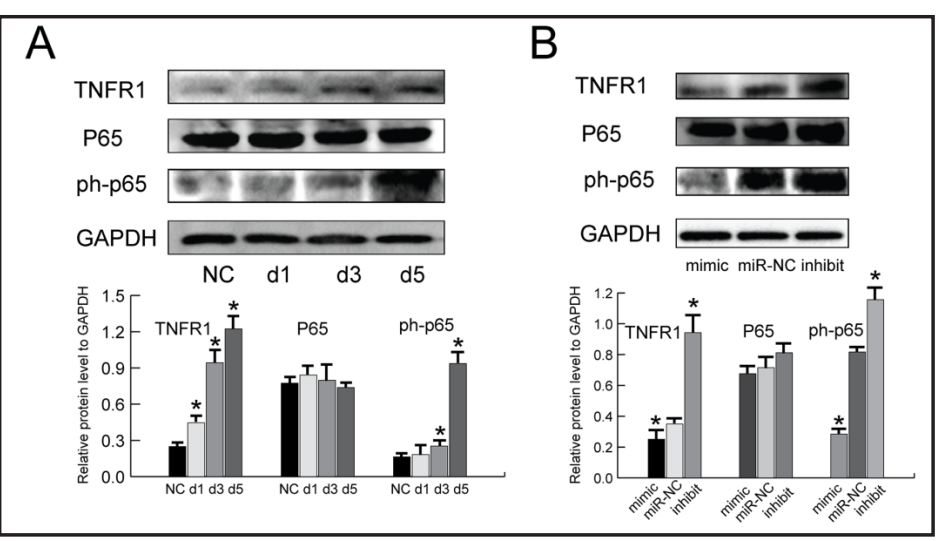

the best of our knowledge, TNF/TNFR1 is an initiator of nuclear factor- $\kappa \mathrm{B}$ (NF- $\kappa \mathrm{B}$ ) signaling pathway activation, which is closely associated with osteoclastogenesis. We first detected TNFR1 expression and p65 phosphorylation levels on day 1, 3, and 5 after osteoclastogenesis. Levels of TNFR1 and phosphorylated p65 (ph-p65) significantly increased after RANKL induction (Fig. 4A). Further, we investigated whether miR-218 attenuated RAW 264.7 cell osteoclastic differentiation by targeting NF- $\kappa B$ signaling. To this end, we investigated the protein levels of p65, which is the TNFR1 downstream molecule closely related to osteoclastogenesis. miR-218 overexpression and inhibition did not significantly alter total p65 levels. We also evaluated ph-p65 levels; western blotting revealed markedly increased ph-p65 expression when miR-218 expression levels were low, whereas miR-218 overexpression remarkably decreased ph-p65 levels. The abovementioned results indicate that miR-218 inhibits osteoclast differentiation by suppressing the p65 pathway (Fig. 4B).

\section{Discussion}

Our results showed that miR-218 is a negative regulator of osteoclastogenesis and is downregulated during RAW 264.7 cell osteoclastic differentiation. miR-218 overexpression attenuated osteoclast differentiation, whereas inhibiting miR-218 activated osteoclastogenesis. Luciferase reporter assay identified Tnfr1 as a direct target of miR-218. To further investigate the mechanism underlying miR-218 regulation of osteoclastogenesis, we investigated the TNF signaling pathway. We found that low miR-218 expression promoted osteoclastogenesis by targeting TNFR1 and activating the NF- $\kappa$ B pathway, which reveals the importance of miR-218 in the development of postmenopausal osteoporosis. Excessive osteoclast differentiation leads to osteoporosis; therefore, inhibiting osteoclast differentiation may alleviate osteoporosis [2, 3].

TNFR1 is a cell membrane receptor of TNF, which belongs to the TNF receptor superfamily $[21,22]$. TNF signals through two cell surface receptors: TNFR1 and TNFR2. Multiple experimental methods have shown that TNFR1 initiates most of the biological activity of TNF. The binding of TNF to TNFR1 elicits a series of intracellular events [23]. Many published studies have described the role of TNF in the development of osteoporosis. For example, TNF/TNFR1 signaling promoted osteoclastogenesis by JNK signaling-induced expression of semaphorin 3D [24]. Another study showed that TNF/TNFR1 signaling suppressed bone formation in estrogen deficiency-induced osteoporosis by inhibiting semaphorin 3B via Wnt/ $\beta$-catenin signaling [25]. Gerstenfeld et al. [26] suggested that TNF/ TNFR1 signaling plays a key role in promoting bone repair by inducing bone progenitor recruitment or osteoblast activation in the context of endochondral bone formation.

TNF activates NF- $\mathrm{BB}$ [27-29]. Signal transduction of the TNFR superfamily member activates TAK1, which induces activation of the I $\mathrm{B}$ kinase complex, thereby activating $\mathrm{NF}-\kappa \mathrm{B}[30]$. NF- $\kappa \mathrm{B}$ is a multivalent transcription factor involved in regulating osteoclast 


\section{Cellular Physiology \begin{tabular}{l|l|l} 
DOI: $10.1159 / 000491740$ & $\begin{array}{l}\text { C } 2018 \text { The Author(s). Published by S. Karger AG, Basel } \\
\text { www.karger.com/cpb }\end{array}$
\end{tabular}

formation, function, and survival [31]. It regulates early osteoclast differentiation by activating c-Fos and NFATC1 [32, 33]. The NF- $\kappa B$ complex has five original members: RelA (p65), p50, p52, RelB, and c-Rel. p65 preferentially dimerizes with p50 and produces signals in the classic pathways [34]. p65 promotes osteoporosis differentiation by blocking a RANKL-induced, apoptotic JNK-Bid pathway [35], participates in osteoclast apoptosis, and is associated with bone homeostasis [36, 37].

In this study, we found that miR-218 overexpression downregulated TNFR1, thereby inhibiting TNF signaling and consequently hindering activation of p-p65 (Fig. 5), which is consistent with previous studies [33, 37-39]. Therefore, we suggest that miR-218 overexpression may attenuate osteoclast differentiation, after which the NF- $\kappa$ B pathway decreases bone mass loss caused by osteoclasts, and that miR-218 may delay the progress of postmenopausal osteoporosis. Reduced osteoclast bone resorption may decrease fracture risk in postmenopausal osteoporosis [40, 41]; thus, targeting miR-218 may be a potential therapeutic option for postmenopausal osteoporosis.

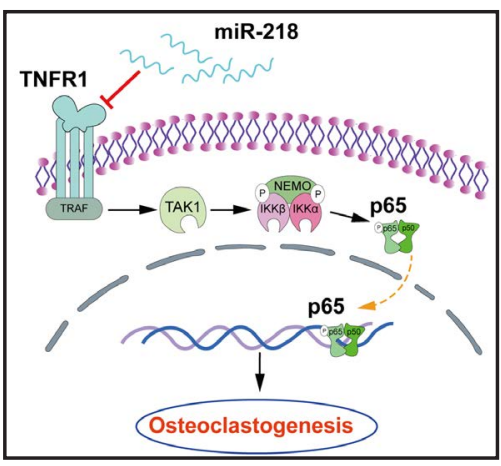

Fig. 5. Possible mechanisms of miR-218 regulation of osteoclast differentiation. miR-218 downregulation upregulated TNFR1, activating TNF signaling. The signal transduction of the TNFR superfamily member activated TAK1, which induced IKK activation, thereby activating $\mathrm{NF}-\kappa \mathrm{B}$ and resulting in the osteoclastic differentiation of RAW 264.7 cells.

To the best of our knowledge, this is the first study to investigate miR-218-regulated osteoclastogenesis via suppressing the NF- $\kappa B$ signaling pathway by targeting TNFR1. However, a main limitation of this study was that we evaluated the underlying regulatory mechanisms of miR-218 in osteoclastogenesis in only in vitro experiments. Thus, future studies are needed to confirm out results in vivo.

\section{Conclusion}

Our results highlight the importance of miR-218 in regulating osteoclastogenic differentiation by targeting TNFR1. Furthermore, our findings provide a theoretical basis for exploring miR-218 as a potential effective therapeutic target in postmenopausal osteoporosis.

\section{Acknowledgements}

Our research done in the manuscript is supported by the National Natural Science Foundation of China (Grant No. 81472989 to Hongxiu Zhang).

\section{Disclosure Statement}

The authors declare that they have no competing interests.

\section{References}

1 Andreopoulou P, Bockman RS: Management of postmenopausal osteoporosis. Annu Rev Med 2015;66:329342 .

-2 Black DM, Rosen CJ: Postmenopausal Osteoporosis. N Engl J Med 2016;374:2096-2097. 


\section{Cellular Physiology Cell Physiol Biochem 2018;48:339-347 \begin{tabular}{ll|l} 
DOI: 10.1159/000491740 & Ond Biochemistry 2018 The Author(s). Published by S. Karger AG, Basel \\
wwww.karger.com/cpb
\end{tabular}

- Emkey GR, Epstein S: Secondary osteoporosis: pathophysiology \& diagnosis. Best Pract Res Clin Endocrinol Metab 2014;28:911-935.

-4 Yang L, Ge D, Cao X, Ge Y, Chen H, Wang W, Zhang H: MiR-214 Attenuates Osteogenic Differentiation of Mesenchymal Stem Cells via Targeting FGFR1 Cell Physiol Biochem 2016;38:809-820.

5 Fabian MR, Sonenberg N, Filipowicz W: Regulation of mRNA translation and stability by microRNAs. Annu Rev Biochem 2010;79:351-379.

-6 Galasso M, Sana ME, Volinia S: Non-coding RNAs: a key to future personalized molecular therapy? Genome Med 2010;2:12.

-7 Ghelani HS, Rachchh MA, Gokani RH: MicroRNAs as newer therapeutic targets: A big hope from a tiny player. J Pharmacol Pharmacother 2012;3:217-227.

-8 Li Y, Kowdley KV: MicroRNAs in common human diseases. Genomics Proteomics Bioinformatics 2012;10:246-253.

-9 van Wijnen AJ, van de Peppel J, van Leeuwen JP, Lian JB, Stein GS, Westendorf JJ, Oursler MJ, Im HJ, Taipaleenmaki H, Hesse E, Riester S, Kakar S: MicroRNA functions in osteogenesis and dysfunctions in osteoporosis. Curr Osteoporos Rep 2013;11:72-82.

10 Gennari L, Bianciardi S, Merlotti D: MicroRNAs in bone diseases. Osteoporos Int 2017;28:1191-1213.

11 Yuan Y, Zhang L, Tong X, Zhang M, Zhao Y, Guo J, Lei L, Chen X, Tickner J, Xu J, Zou J: Mechanical Stress Regulates Bone Metabolism Through MicroRNAs. J Cell Physiol 2017;232:1239-1245.

12 Hao L, Li J, Tian Y, Wu J: Changes in the MicroRNA Profile of the Mandible of Ovariectomized Mice. Cell Physiol Biochem 2016;38:1267-1287.

13 Ji X, Chen X, Yu X: MicroRNAs in Osteoclastogenesis and Function: Potential Therapeutic Targets for Osteoporosis. Int J Mol Sci 2016;17:349.

14 Hassan MQ Maeda Y, Taipaleenmaki H, Zhang W, Jafferji M, Gordon JA, Li Z, Croce CM, van Wijnen AJ, Stein JL, Stein GS, Lian JB: miR-218 directs a Wnt signaling circuit to promote differentiation of osteoblasts and osteomimicry of metastatic cancer cells. J Biol Chem 2012;287:42084-42092.

15 Zhang WB, Zhong WJ, Wang L: A signal-amplification circuit between miR-218 and Wnt/beta-catenin signal promotes human adipose tissue-derived stem cells osteogenic differentiation. Bone 2014;58:59-66.

-16 Zhang Y, Xie RL, Croce CM, Stein JL, Lian JB, van Wijnen AJ, Stein GS: A program of microRNAs controls osteogenic lineage progression by targeting transcription factor Runx2 Proc Natl Acad Sci U S A 2011;108:9863-9868.

17 Chen C, Cheng P, Xie H, Zhou HD, Wu XP, Liao EY, Luo XH: MiR-503 regulates osteoclastogenesis via targeting RANK. J Bone Miner Res 2014;29:338-347.

18 Wu Q, Zhou X, Huang D, Ji Y, Kang F: IL-6 Enhances Osteocyte-Mediated Osteoclastogenesis by Promoting JAK2 and RANKL Activity In Vitro. Cell Physiol Biochem 2017;41:1360-1369.

19 Muruganandan S, Dranse HJ, Rourke JL, McMullen NM, Sinal CJ: Chemerin neutralization blocks hematopoietic stem cell osteoclastogenesis. Stem Cells 2013;31:2172-2182.

20 Tang L, Yin Y, Liu J, Li Z, Lu X: MiR-124 Attenuates Osteoclastogenic Differentiation of Bone Marrow Monocytes Via Targeting Rab27a. Cell Physiol Biochem 2017;43:1663-1672.

21 Locksley RM, Killeen N, Lenardo MJ: The TNF and TNF receptor superfamilies: integrating mammalian biology. Cell 2001;104:487-501.

$>22$ Idriss HT, Naismith JH: TNF alpha and the TNF receptor superfamily: structure-function relationship(s). Microsc Res Tech 2000;50:184-195.

-23 Chen G, Goeddel DV: TNF-R1 signaling: a beautiful pathway. Science 2002;296:1634-1635.

-24 Sang C, Zhang J, Zhang Y, Chen F, Cao X, Guo L: TNF-alpha promotes osteoclastogenesis through JNK signaling-dependent induction of Semaphorin3D expression in estrogen-deficiency induced osteoporosis. J Cell Physiol 2017;232:3396-3408.

25 Sang C, Zhang Y, Chen F, Huang P, Qi J, Wang P, Zhou Q Kang H, Cao X, Guo L: Tumor necrosis factor alpha suppresses osteogenic differentiation of MSCs by inhibiting semaphorin 3B via Wnt/beta-catenin signaling in estrogen-deficiency induced osteoporosis. Bone 2016;84:78-87.

26 Gerstenfeld LC, Cho TJ, Kon T, Aizawa T, Cruceta J, Graves BD, Einhorn TA: Impaired intramembranous bone formation during bone repair in the absence of tumor necrosis factor-alpha signaling. Cells Tissues Organs 2001;169:285-294.

27 Hsu H, Xiong J, Goeddel DV: The TNF receptor 1-associated protein TRADD signals cell death and NF-kappa B activation. Cell 1995;81:495-504. 


\section{Cellular Physiology Cell Physiol Biochem 2018:48:339-347 \begin{tabular}{l|l} 
DOI: 10.1159/000491740 & $\begin{array}{l}\text { O 2018 The Author(s). Published by S. Karger AG, Basel } \\
\text { www.karger.com/cpb }\end{array}$
\end{tabular} \\ Wang et al.: MiR-218 Downregulation of Osteoclastogenesis}

28 Hayden MS, Ghosh S: Signaling to NF-kappaB. Genes Dev 2004;18:2195-2224.

29 Mitchell S, Vargas J, Hoffmann A: Signaling via the NFkappaB system. Wiley Interdiscip Rev Syst Biol Med 2016;8:227-241.

30 Hayden MS, West AP, Ghosh S: SnapShot: NF-kappaB signaling pathways. Cell 2006;127:1286-1287.

-31 Soysa NS, Alles N: NF-kappaB functions in osteoclasts. Biochem Biophys Res Commun 2009;378:1-5.

-32 Yamashita T, Yao Z, Li F, Zhang Q Badell IR, Schwarz EM, Takeshita S, Wagner EF, Noda M, Matsuo K, Xing L, Boyce BF: NF-kappaB p50 and p52 regulate receptor activator of NF-kappaB ligand (RANKL) and tumor necrosis factor-induced osteoclast precursor differentiation by activating c-Fos and NFATc1 J Biol Chem 2007;282:18245-18253.

-33 Boyce BF, Yao Z, Xing L: Functions of nuclear factor kappaB in bone. Ann N Y Acad Sci 2010;1192:367-375.

-34 Boyce BF, Xiu Y, Li J, Xing L, Yao Z: NF-kappaB-Mediated Regulation of Osteoclastogenesis. Endocrinol Metab (Seoul) 2015;30:35-44.

35 Vaira S, Alhawagri M, Anwisye I, Kitaura H, Faccio R, Novack DV: RelA/p65 promotes osteoclast differentiation by blocking a RANKL-induced apoptotic JNK pathway in mice. J Clin Invest 2008;118:20882097.

-36 Novack DV: Unique personalities within the NF-kappaB family: distinct functions for p65 and RelB in the osteoclast. Adv Exp Med Biol 2011;691:163-167.

37 Mise-Omata S, Alles N, Fukazawa T, Aoki K, Ohya K, Jimi E, Obata Y, Doi T: NF-kappaB RELA-deficient bone marrow macrophages fail to support bone formation and to maintain the hematopoietic niche after lethal irradiation and stem cell transplantation. Int Immunol 2014;26:607-618.

-38 Oeckinghaus A, Hayden MS, Ghosh S: Crosstalk in NF-kappaB signaling pathways. Nat Immunol 2011;12:695-708.

-39 Teitelbaum SL, Ross FP: Genetic regulation of osteoclast development and function. Nat Rev Genet 2003;4:638-649.

-40 Cosman F, Crittenden DB, Adachi JD, Binkley N, Czerwinski E, Ferrari S, Hofbauer LC, Lau E, Lewiecki EM, Miyauchi A, Zerbini CA, Milmont CE, Chen L, Maddox J, Meisner PD, Libanati C, Grauer A: Romosozumab Treatment in Postmenopausal Women with Osteoporosis. N Engl J Med 2016;375:1532-1543.

41 Gennari L, Rotatori S, Bianciardi S, Nuti R, Merlotti D: Treatment needs and current options for postmenopausal osteoporosis. Expert Opin Pharmacother 2016;17:1141-1152. 